\title{
УДК 615.014.21:54.062
}

\section{ВАЛИДАЦИЯ МЕТОДИКИ КОЛИЧЕСТВЕННОГО ОПРЕДЕЛЕНИЯ ФЕНОБАРБИТАЛА ПРИ ПРОВЕДЕНИИ ТЕСТА «РАСТВОРЕНИЕ» В ТАБЛЕТКЕ СУБЛИНГВАЛЬНОЙ «КОРВАЛОЛ»}

\author{
() И. О. Омельченко, Т. Г. Ярных, Г. И. Борщевський, В. А. Коноваленко
}

Проведена валидация методики количественного определения фенобарбитала при проведении теста
«Растворение» методом высокоэффективной жидкостной хроматографии в таблетках сублингваль-
ных «Корвалол».
Цель: провести валидацию методики количественного определения фенобарбитала при проведении те-
ста «Растворение» в соответствии с требованиями Государственной Фармакопеи Украины (ГФУ);
при помощзи экспериментальных исследований доказать, что методика позволяет достоверно контро-
лировать количественное содержание фенобарбитала в таблетке сублингвальной «Корвалол».
Методы: исследование проводилось методом ВЭЖХв соответствии с требованиями ГФУ.
Результаты: было проведено изучение критериев специфичности методики количественного определе-
ния фенобарбитала, ее правильности, прецизионности, линейности и робастности. Установлено, что
методика соответствует требованиям ГФУ по основным валидационным показателям. Результатьт
исследований использованы при разработке методов контроля качества таблеток сублинвальных «Ко-
рвалол».

Выводы: проведено валидацию методики количественного контроля фенобарбитала при проведении теста «Растворение» для препарата «Корвалол», таблетки сублингвальные в соответствии с требованиями ГФУ. Доказано, что методика соответствует требованиям ГФУ по специфичности, правильности, прецизионности и робастности в диапазоне 60-120\% от номинального содержания. Полученнье результаты использованы при разработке методов контроля качества в препарате «Корвалол» таблетки сублингвальные

Ключевые слова: валидация, специфичность, правильность, прецизионность, робастность, хроматография, растворение, фенобарбитал, сублингвальные таблетки

Validation of method for quantitative determination of Phenobarbital in the sublingual tablet "Corvalol" at "Dissolution" test was carried out by high performance liquid chromatography method.

Aim. To validate the method for quantitative determination of Phenobarbital at "Dissolution" test in accordance with the State Pharmacopoeia of Ukraine (SPhU) requirements; to prove by experimental research that the method allows reliably monitoring Phenobarbital content in the sublingual tablet "Corvalol".

Methods. HPLC method was used for research in accordance with the SPhU requirements.

Results. The study of specificity criteria of method for Phenobarbital quantitative determination, its correctness, precision, linearity and robustness was carried out. It was determined, that the method meets the SPhU requirements on the main validation parameters. Results of research were used for development of methods for quality control of sublingual tablets "Corvalol".

Conclusion. Validation of the method for quantitative determination of Phenobarbital in the remedy "Corvalol" sublingual tablets at "Dissolution" test in accordance with the State Pharmacopoeia of Ukraine (SPhU) requirements was carried out. It has been proven that the method meets the SPhU requirements on the specificity, correctness, precision, and robustness in the range of 60-120\% of the nominal content. The obtained data were used for development of methods for quality control of the remedy "Corvalol" sublingual tablets

Keywords: validation, specificity, correctness, precision, robustness, chromatography, dissolution, Phenobarbital, sublingual tablets

\section{1. Введение}

Актуальной задачей современной фармацевтики является поиск, создание и стандартизация, как новых лекарственных препаратов, так и других форм уже существующих лекарственных препаратов. Особое внимание уделяется разработке и стандартизации препаратов в твердой форме аналогичных по составу и действию уже существующих в жидкой форме. Это обуславливается тем, что при современном ритме жизни пациенты отдают предпочтение приему лекарственных препаратов в твердой форме [1].
2. Постановка проблемы в общем виде, актуальность темы и ее связь с важными научными или практическими вопросами

На сегодняшний день возникают трудности с анализом отдельных компонентов лекарственных препаратов вследствие взаимного влияния на результаты анализа вспомогательных веществ, которые присутствуют в твердой лекарственной форме и отсутствуют, например, в жидкой. Поэтому большое значение для методик анализа имеет пробоподготовка, которая позволяет нивелировать влияние отдельных компонентов. 


\section{3. Анализ последних исследований и публи-} каций, в которых начато решение данной проблемы и на которые опирается автор

В представленной работе объектом исследования выбрано разработанную нами таблетку «Корвалол». Состав таблеток «Корвалол» полностью соответствует «Корвалолу» в форме капель, при этом одна таблетка является разовой дозой на один прием. Корвалол таблетки можно принимать сублингвально - это обеспечивает быстрое наступление эффекта, уже через 5-10 минут, и не нуждается в жидкости для запивания. Одна таблетка содержит 12,42 мг этилового эфира альфа-бромизовалериановой кислоты, 11,34 мг фенобарбитала, 0,88 мг масла мяты, вспомагательными веществами являются лактоза моногидрат, магния стеарат, $\beta$-циклодекстрин, калия ацесульфам. Одним из важнейших тестов для сублингвальных таблеток является тест «Растворение», так как скорость поступления действующих веществ в кровоток пациента напрямую зависит от скорости растворения препарата в ротовой полости [2-4].

4. Выделение не решенных ранее частей общей проблемы, которой посвящена статья

Несмотря на значительное количество работ, посвящённых количественному определению фенобарбитала, не были проведены исследования, непосредсвенно, для сублингвальных таблеток «Корвалол». Проведение данных исследований необходимо для разработки методов контроля качества данного лекарственного препарата [5, 6].

\section{5. Формулирование целей (задач) статьи}

Целью данной работы является проведение валидации методики количественного определения фенобарбитала при проведении теста «Растворение» в соответствии с требованиями ГФУ $[7,8]$. При помощи экспериментальных исследований необходимо доказать, что методика позволяет достоверно контролировать количественное содержание фенобарбитала в таблетке сублингвальной «Корвалол» при проведении теста «Растворение».

\section{6. Изложение основного материала исследо-} вания (методов и объектов) с обоснованием полученных результатов

В работе был использован фенобарбитала стандартный образец ПАТ «Фармак» (с.AVA0381), вода для хроматографии (ф. ПАТ «Фармак», Украина), метанол для хроматографии (ф. Sigma-Aldrich $\mathrm{GmbH}$, Германия), уксусная кислота (ф. Sigma-Aldrich $\mathrm{GmbH}$, Германия), натрия ацетат тригидрат (ф. Sigma-Aldrich $\mathrm{GmbH}$, Германия).

Приборы: Тест растворения проводили на приборе ERWEKA (Германия), анализ полученных проб проводился на жидкостном хроматографе Agilent1200 (США), взвешивание проводилось на аналитических весаx Sartorius ED124S (Германия). В работе использована лабораторная посуда класса А, которая отвечает ГСТУ 29228-91 [9].

Методика количественного определения: определение проводится методом жидкостной хро- матографии в соответствии с требованиями ГФУ [10]. Растворение проводилось на приборе с лопастью, среда растворения вода. Одну таблетку помещают в посудину для растворения и при оборотах лопасти 75 об/мин растворяют 30 минут. Через 30 минут из центра посудины отбирают 25 мл раствора, фильтруют сквозь мембранный фильтр с диаметром пор 0,45 мкм, отбрасывая первые пять миллилитров фильтрата.

Хроматографирование проводили при следующих условиях:

- подвижная фаза ацетатный буфер $\mathrm{pH} 4.5$, Р - метанол (65:35);

- скорость подвижной фазы 1,0 мл/мин;

- температура колонки $45{ }^{\circ} \mathrm{C}$;

- детектирование при длине волны 220 нм;

- колонка октадецилсилильная размером 4,6× $\times 50$ мм, размер частиц сорбента 5 мкм;

Хроматографируют полученный тестовый раствор и раствор сравнения, в котором содержится 0,0132 мг/мл фенобарбитала.

Хроматографическая система считается пригодной, если выполняются следующие условия:

Эффективность хроматографической колонки, рассчитанная для пиков фенобарбитала, должна быть не менее 1000 теоретических тарелок;

Коэффициент симметрии пика фенобарбитала должен быть не более 1,5;

Относительное стандартное отклонение для площадей пиков фенобарбитала не должно превышать $2 \%$, рассчитанное для результатов трех инжекций.

Количество фенобарбитала, которое перешло из таблетки в раствор, в процентах, рассчитывают по формуле:

$$
X=\frac{S_{1} \times m_{0} \times P \times 0.0054 \times\left(100-W_{0}\right)}{S_{0} \times b \times n},
$$

где $\mathrm{S}_{0}$ - среднее значение площадей пиков фенобарбитала, полученное с хроматограмм раствора сравнения;

$\mathrm{S}_{1}$ - среднее значение площадей пиков фенобарбитала, полученное с хроматограмм тестового раствора;

$\mathrm{m}_{0}$ - масса навески фенобарбитала, взятого для приготовления раствора сравнения, в граммах;

b - содержание фенобарбитала в таблетке, в граммах;

P - содержание основного вещества в стандартном образце фенобарбитала в пересчете на сухое вещество, в процентах;

$\mathrm{W}_{0}$ - содержание воды в стандартном образце фенобарбитала, в процентах;

Препарат удовлетворяет требованием, если степень растворения фенобарбитала (Q) испытуемых таблеток соответствует требованиям ГФУ, 2.9.3 (таблица 2.9.3-1) и через 30 минут должна быть не менее $85 \%$ от содержания в одной таблетке.

Для оценки метрологических характеристик методики предварительно были рассчитаны критерии приемлемости в соответствии с требованиями ГФУ[4], представленные в табл. 1. 
Критерии приемлемости метрологических характеристик методики

\begin{tabular}{|c|c|}
\hline Критерии приемлемости метрологических характеристик & Величина критических значений, \% \\
\hline Максимально допустимая полная неопределенность методики $-\max \Delta \mathrm{As}$ & 0.96 \\
\hline Максимальная неопределенность при пробоподготовке $-\max \Delta \mathrm{Sp}$ & 2,10 \\
\hline Критическое значение стандартного отклонения - RSD0 & 0,99807443 \\
\hline Индекс кореляции - Rс & 2.6190654 \\
\hline Критическое практически незначительное значение свободного члена - a
\end{tabular}

Пригодность методики оценивали по следующим параметрам:

- Специфичность

- Линейность в диапазоне 60-120\% от номинальной концентрации

- Прецизионность

- Правильность

- Граница количественного определения

- Робастность

Для подтверждения специфичности методики на хроматограмме раствора плацебо должны отсутствовать пики, которые совпадают по времени удержания с временем удержания пика фенобарбитала на хроматограмме раствора сравнения. В случае присутствия таких пиков их площадь должна быть незначительной в сравнении с максимально допустимой неопределенностью результатов пробоподготовки: $\max \Delta \mathrm{As} * 0,32=0,96 \%$, их площадь не должна превышать $0,96 \%$ от средней площади пиков фенобарбитала на хроматограммах растворов сравнения. На хроматограммах растворов плацебо пики, которые совпадают по времени удержания со временем удержания пика фенобарбитала на хроматограмме раствора сравнения отсутствуют, следовательно, критерии специфичности методики выдерживаются.

Раствор сравнения готовили согласно аналитической методике, раствор плацебо готовили аналогично раствору сравнения.

Для проверки правильности и прецизионности методики готовили три отдельных смеси с точно известным содержанием фенобарбитала, которые охватывали диапазон применения методики (с концентрациями $60 \%, 100 \%$ и $120 \%$ от номинальной). Для каждой модельной смеси было проведено три параллельных анализа, в соответствии с требованиями ГФУ рассчитывали следующие критерии: систематическая погрешность $\delta$ \% (для правильности) и относительный доверительный интервал $\Delta \mathrm{z}$ (для прецизионности):

$$
\begin{gathered}
S_{z}(\%)=\sqrt{\frac{\sum_{i=1}^{n}\left(Z_{i}-\bar{Z}\right)^{2}}{n-1}} ; \\
\Delta_{\mathrm{z}}=S_{z}(\%) \cdot t(95 \%, n-1) \leq \Delta_{A s} ; \\
\delta \%=|\bar{Z}-100| \leq \frac{\Delta_{Z}}{\sqrt{n}},
\end{gathered}
$$

где $\mathrm{S}_{\mathrm{z}}$ - относительное стандартное отклонение, \% (рассчитанное для соотношений «введено/найдено»); $\mathrm{n}$ - объем выборки;

$\Delta \mathrm{z}$ - относительный доверительный интервал; $\Delta \mathrm{A}_{\mathrm{s}}-$ Критическое значение неопределенности методики;

$\mathrm{t}$ - односторонний критерий Стьюдента для вероятности $95 \%$ и числа степеней свободы v=n-1;

$\delta \%$ - систематическая погрешность;

$\mathrm{Z}$ - найденное содержание, в \% до введенного;

$\mathrm{Z}$ - среднее значение Z.

Результаты исследований и приведенных расчётов приведены в табл. 2 .

Как видно с табл. 2, экспериментальные результаты прецизионности характеризуются допустимым отклонением относительно среднего значения и соответственно низким стандартным отклонением $\mathrm{Sz} \%(\mathrm{Sz} \%=0,961<3,0)$ во всем диапазоне концентраций (60-20\%), что позволяет говорить о качестве работы анализируемой аналитической методики. Систематическая погрешность методики $\delta \%=0,713$, что характеризует достаточную близость среднего результата полученной оптической плотности растворов к его номинальному значению. Критерии правильности и прецизионности методики определения фенобарбитала выдерживаются.

Для изучения линейности готовились семь растворов с разными концентрациями фенобарбитала в диапазоне от $60 \%$ до $120 \%$ от номинальной концентрации, растворы анализировали с помощью предложенного метода. На основе определённых площадей пиков растворов с точно известной концентрацией фенобарбитала построили график зависимости площади пика от концентрации у нормализованных координатах и рассчитывали параметры линейности. Полученные результаты и их статистическая обработка приведены в табл. 3 .

На основании полученных данных можно сделать вывод, что линейности методики подтверждается во всем диапазоне исследованных концентраций: коэффициент корреляции равен 0,9995169, остаточное стандартное отклонение равно 0,74600347. График зависимости площади пика от концентрации проходит практически через начало координат (точка пересечения с осью ординат - 1,5754155), рассчитанный доверительный интервал константы $\Delta$ а меньше модуля самой константы, что свидетельствует о ее статистической и практической незначительности.

График и уравнение линейной зависимости приведены на рис. 1. 
Таблица 2

Результаты исследования правильности и прецизионности методики определения фенобарбитала

\begin{tabular}{|c|c|c|c|}
\hline $\begin{array}{c}\text { Содержание в модельной смеси, } \\
\text { \% от номинального }\end{array}$ & $\begin{array}{l}\text { Площадь пика } \\
\text { фенобарбитала }\end{array}$ & $\begin{array}{c}\text { Найденное содержание до } \\
\text { номинального, \% }\end{array}$ & $\begin{array}{c}\text { Найденное содержание до } \\
\text { введенного, \% }\end{array}$ \\
\hline \multirow{3}{*}{60} & 708,8 & 60,598 & 99,63 \\
\hline & 704,3 & 60,213 & 101,065 \\
\hline & 692,3 & 59,187 & 101,81 \\
\hline \multirow{3}{*}{100} & 1172,7 & 98,673 & 99,562 \\
\hline & 1173,2 & 98,715 & 101,227 \\
\hline & 1219,5 & 102,611 & 101,71 \\
\hline \multirow{3}{*}{120} & 1431,8 & 120,28 & 101,39 \\
\hline & 1413,7 & 118,759 & 98,97 \\
\hline & 1439,9 & 120,96 & 101,41 \\
\hline \multicolumn{3}{|l|}{ Среднее значение Z } & 100,75 \\
\hline \multicolumn{3}{|c|}{ Относительное стандартное отклонение Sz } & 0,961 \\
\hline \multicolumn{3}{|c|}{ Относительный доверительный интервал $\Delta \mathrm{z}$} & 1,8667611 \\
\hline \multicolumn{3}{|c|}{ Критическое значение неопределенности методики $\Delta \mathrm{As}$} & $<3,0$ \\
\hline \multicolumn{3}{|c|}{ Систематическая погрешность $\delta \%$} & 0,713 \\
\hline \multicolumn{3}{|c|}{ Критерий неопределённости систематической погрешности } & $<0,948$ \\
\hline
\end{tabular}

Результаты исследования линейности методики определения фенобарбитала

Таблица 3

\begin{tabular}{|c|c|c|c|c|c|}
\hline $\begin{array}{c}\text { Концентрация, } \\
\text { мг/мл }\end{array}$ & $\begin{array}{l}\text { Концен- } \\
\text { трация, \% }\end{array}$ & $\begin{array}{l}\text { Площадь } \\
\text { пика }\end{array}$ & $\begin{array}{c}\text { Площадь пика, \% от } \\
\text { номинальной }\end{array}$ & \multicolumn{2}{|c|}{$\begin{array}{c}\text { Критериальные требования и } \\
\text { приемлимость }\end{array}$} \\
\hline 0,00798 & 60 & 701,8 & 59,775 & \multirow{7}{*}{\multicolumn{2}{|c|}{$\begin{array}{c}\text { Уравнение прямой: } \\
\text { Sнорм=1,0275741* Снорм-1,5754155 } \\
\text { Тангенс угла наклона: } \mathrm{b}=1,0275741 \\
\text { Точка пересечения с осью ординат: } \\
\mathrm{a}=-1,5754155 \\
\text { Коэффициент корреляции: } \\
=0,9995169\end{array}$}} \\
\hline 0,00931 & 70 & 830,6 & 70,746 & & \\
\hline 0,01064 & 80 & 956,23 & 81,446 & & \\
\hline 0,01197 & 90 & 1052,033 & 89,606 & & \\
\hline 0,0133 & 100 & 1188,467 & 101,227 & & \\
\hline 0,01463 & 110 & 1313,5 & 111,876 & & \\
\hline 0,01596 & 120 & 1428,467 & 121,668 & & \\
\hline \multicolumn{4}{|c|}{ Коэффициент корреляции: r=0,9995169 } & $\mathrm{r}>0,99807443$ & выдерживает \\
\hline \multicolumn{4}{|c|}{$\begin{array}{l}\text { Остаточная дисперсия: RSD02 }=0,55652118 \\
\text { Остаточное стандартное отклонение: RSD0=0,74600347 }\end{array}$} & $\begin{array}{c}\text { RSD0/b } \leq \\
\leq 1,4888337\end{array}$ & выдерживает \\
\hline \multicolumn{4}{|c|}{$\begin{array}{c}\text { Доверительный интервал константы, а: } \\
\qquad \begin{array}{c}\Delta \mathrm{a}=1,2997843 \\
\mathrm{a}=-1.5754155\end{array}\end{array}$} & $\begin{array}{c}<\mathrm{IaI} \\
\leq 2.6190654\end{array}$ & $\begin{array}{c}\text { Выдерживает по } \\
\text { критерию стати- } \\
\text { стической и практи- } \\
\text { ческой незначи- } \\
\text { тельности } \\
\end{array}$ \\
\hline
\end{tabular}

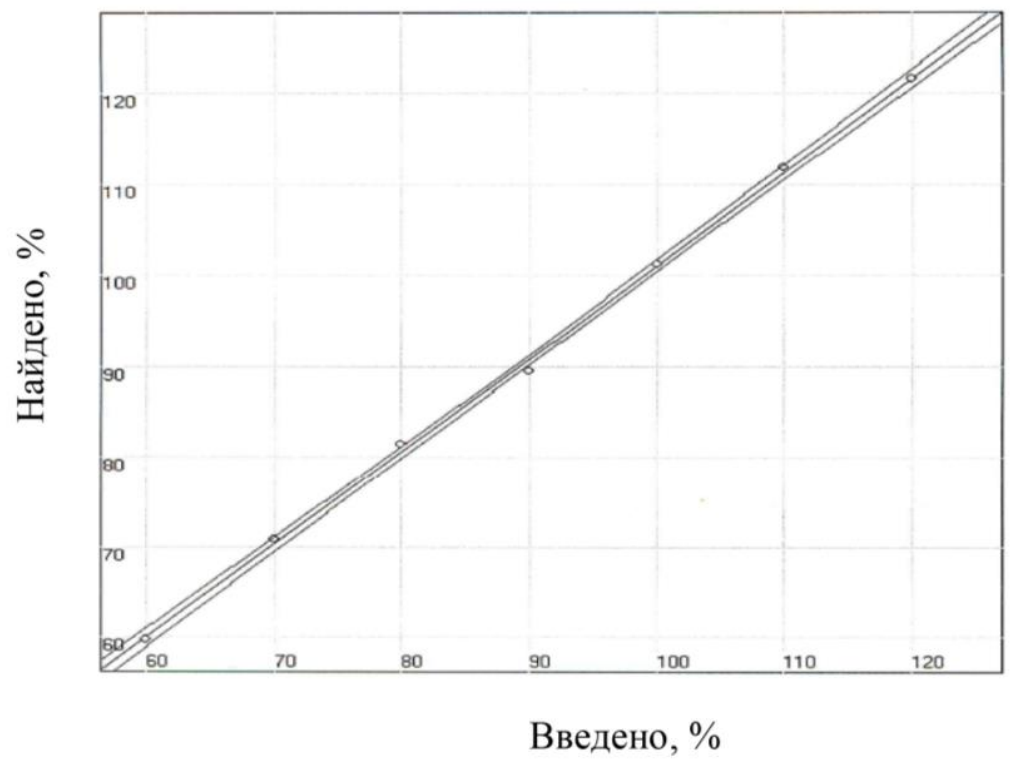

Рис. 1. График зависимости площади пиков от концентрации фенобарбитала в нормализованных координатах 
Робастность - устойчивость методики к небольшим изменениям условий эксперимента проверяли на исследуемом растворе. Условия хроматографирования изменялись в границах $\pm 10 \%$ от указан ных в методике, при этом рассматривали такие параметры хроматограммы как коэффициент симметрии пиков и число теоретических тарелок.

Результаты исследований приведены в табл. 4.

Таблица 4

Результаты исследования робастности методики количественного определения фенобарбитала для теста «Растворение» в препарате «Корвалол» таблетки сублингвальные

\begin{tabular}{|c|c|c|c|}
\hline \multirow{2}{*}{ Условия проведения анализа } & \multicolumn{3}{|c|}{ Параметры } \\
\cline { 2 - 4 } & $\begin{array}{c}\text { Время удержива- } \\
\text { ния, мин. }\end{array}$ & $\begin{array}{c}\text { Число теоретических } \\
\text { тарелок }\end{array}$ & $\begin{array}{c}\text { Коэффициент } \\
\text { симметрии }\end{array}$ \\
\hline Стандартные условия методики & 2,827 & 6532 & 0,84 \\
\hline Скорость потока 0,9 мл/мин, $(-10 \%)$ & 3,103 & 6023 & 0,80 \\
\hline Скорость потока 1,1 мл/мин, $(+10 \%)$ & 2,531 & 6984 & 0,87 \\
\hline Температура колонки 49,50 ${ }^{\circ} \mathrm{C}$ & 2,971 & 6312 & 0,83 \\
\hline Температура колонки 40,50 ${ }^{\circ} \mathrm{C}$ & 2,804 & 6101 & 0,88 \\
\hline$+10 \%$ ацетатного буфера & 3,514 & 5458 & 0,84 \\
\hline$+10 \%$ метанола & 2,753 & 6245 & 0,80 \\
\hline
\end{tabular}

Как видно из табл. 4, больше всего на результаты влияют изменения скорости потока подвижной фазы и увеличение массовой доли буферного раствора в ее составе. Также из таблицы видно, что методика устойчива к небольшим изменениям условий эксперимента, что подтверждает ее робастность.

\section{7. Выводы}

Проведено валидацию методики количественного контроля фенобарбитала при проведении теста «Растворение» для препарата «Корвалол», таблетки сублингвальные в соответствии с требованиями ГФУ.

1. Доказано, что методика количественного определения фенобарбитала соответствует требованиям ГФУ по специфичности, правильности, прецизионности и робастности в диапазоне 60-120\% от номинального содержания.

2. Полученные результаты использованы при разработке методов контроля качества в препарате «Корвалол» таблетки сублингвальные.

\section{Литература}

1. Серединская, Н. Н. Корвалол: эффективность и безопасность подтверждаются - значит жизнь препарата продолжается. Сообщение I. Перспективность создания новых лекарственных форм и целесообразность их использования (результаты доклинических исследований) [Текст] / Н. Н. Серединская, М. И. Борщевская, Н. А. Мохорт, Л. М. Киричок // Експериментальна і клінічна медицина. - 2012. - № 4. - С. 22-29.

2. Fu, Y. Orally fast disintegrating tablets: developments, technologies, taste-making and clinical studies [Text] / Y. Fu, S. Yang, S. H. Jeong, S. Kimura, K. Park // Critical Reviews in Therapeutic Drug Carrier Systems. - 2004. - Vol. 21, Issue 6. - P. 433-476. doi: 10.1615/critrevtherdrugcarriersyst. v21.i6.10

3. Nagar, P. Orally disintegrating tablets : formulation, preparation techniques and evaluation [Text] / P. Nagar, K. Singh, I. Chauhan et. al. // Journal of Applied Pharmaceutical Science. - 2011. - Vol. 01, Issue 04. - P. 35-45.

4. Debjit, B. Fast Dissolving Tablet: An Overview [Text] / B. Debjit, B. Chiranjib, R. Krishnakanth et. al. // Journal of Chemical and Pharmaceutical Research. - 2009. - Vol. 1, Issue 1. - P. 163-177.
5. Abdelbary, G. Determination of the in vitro disintegration profile of rapidly disintegrating tablets and correlation with oral disintegration [Text] / G. Abdelbary, C. Eouani, P. Prinderre, J. Joachim, J. Reynier, P. Piccerelle // International Journal of Pharmaceutics. - 2005. - Vol. 292, Issue 1-2. P. 29-41. doi: 10.1016/j.ijpharm.2004.08.019

6. Cirri, M. Development of fast-dissolving tablets of flurbiprofen-cyclodextrin complexes [Text] / M. Cirri, C. Rangoni, F. Maestrelli, G. Corti, P. Mura // Drug Development and Industrial Pharmacy. - 2005. - Vol. 31, Issue 7. - P. 697-707. doi: 10.1080/03639040500253694

7. Гризодуб, А. И. Стандартизованная процедура валидации методик количественного анализа лекарственных средств методом стандарта [Текст] / А. И. Гризодуб, Д. А. Леонтьев, Н. В. Денисенко, Ю. В. Подпружников // Фармаком. - 2004. - № 3. - С. 3-17.

8. Державна фармакопея України [Текст] / Державне підприємство «Науково-експертний фармакопейний центр». - 1-е вид. - Х.: РІРЕГ, 2001. - 556 с.

9. Посуда лабораторная стеклянная. Пипетки градуированные. Ч. 2. Пипетки градуированные без установленного времени ожидания: ГОСТ 29228-91 [Текст]. - М.: Издво стандартов, 1992. -9 с.

10. Державна Фармакопея України. Доповнення 2 [Текст] / Державне підприємство «Науково-експертний фармакопейний центр». - 1-е вид. - Х.: Державне підприємство «Науково-експертний фармакопейний центр», 2008. - 620 с.

\section{References}

1. Seredinskaya, N. N., Borshevskaya, M. I., Mokhort, N. A., Kirichok, L. M. (2012). Corvalol: efficacy and safety are confirmed - mean life of the drug continues. Part I. The prospect of the creation of new dosage forms and the feasibility of their use (preclinical studies). Experimental and clinical medicine, 4, 22-29.

2. Fu, Y., Yang, S., Jeong, S. H., Kimura, S., Park, K. (2004). Orally Fast Disintegrating Tablets: Developments, Technologies, Taste-Masking and Clinical Studies. Critical Reviews in Therapeutic Drug Carrier Systems, 21 (6), 433-476. doi: 10.1615/critrevtherdrugcarriersyst.v21.i6.10

3. Nagar, P., K. Singh, I. Chauhan et. al. (2011). Orally disintegrating tablets : formulation, preparation techniques and evaluation. Journal of Applied Pharmaceutical Science, 01 (04), 35-45.

4. Debjit, B., Chiranjib, B., Krishnakanth, R. et. al. (2009). Fast Dissolving Tablet: An Overview. Journal of Chemical and Pharmaceutical Research, 1 (1), 163-177. 
5. Abdelbary, G., Eouani, C., Prinderre, P., Joachim, J., Reynier, J., Piccerelle, P. (2005). Determination of the in vitro disintegration profile of rapidly disintegrating tablets and correlation with oral disintegration. International Journal of Pharmaceutics, 292 (1-2), 29-41. doi: 10.1016/j.ijpharm. 2004.08.019

6. Cirri, M., Rangoni, C., Maestrelli, F., Corti, G., Mura, P. (2005). Development of Fast-Dissolving Tablets of FlurbiprofenCyclodextrin Complexes. Drug Development and Industrial Pharmacy, 31 (7), 697-707. doi: 10.1080/03639040500253694

7. Grizodub, A. I., Leontiev, D. A., Denisenko, N. V., Podpruzhnikov, Y. V. (2004). A standardized procedure for the validation of quantitative methods of analysis of drugs by standard. Farmakom, 3, 3-17.

8. State Pharmacopoeia of Ukraine (2001). Kharkiv: RIREH, 556.

9. Laboratory glassware. Graduated pipettes. Chep. 2. Graduated pipettes without a specific waiting time: GOST 29228-91 (1992). Moscow: Publishing House of Standards, 9.

10. State Pharmacopoeia of Ukraine. Amendment 2 (2008). Kharkiv: State Enterprise "Scientific-expert Pharmacopoeia Center", 620 .

Дата надходження рукопису 21.09.2016

Омельченко Иларион Александрович, аспирант без отрыва от производства, кафедра технологии лекарств, Национальный фармацевтический университет, ул. Пушкинская, 53, г. Харьков, Украина, 61002; ведущий специалист, лаборатория разработки фармацевтических препаратов департамента по биотехнологии, ПАО «Фармак», ул. Фрунзе, 63, г. Киев, Украина, 04080

E-mail: omelchenko_i@ukr.net

Ярных Татьяна Григорьевна, доктор фармацевтических наук, профессор, Заслуженый деятель науки и техники Украины, кафедра технологии лекарств, Национальный фармацевтический университет, ул. Пушкинская, 53, г. Харьков, Украина, 61002

E-mail: tl.@nuph.edu.ua

Борщевский Геннадий Ильич, доктор фармацевтических наук, начальник лаборатории, лаборатория разработки фармацевтических препаратов департамента по биотехнологии, ПАО «Фармак», ул. Фрунзе, 63, г. Киев, Украина, 04080

Коноваленко Вадим Анатольевич, начальник, Сектор департамента по биотехнологии, ПАО «Фармак», ул. Фрунзе, 63, г. Киев, Украина, 04080

УДК 615.244:614.27

\section{АНАЛИЗ АССОРТИМЕНТА И ДОСТУПНОСТИ ГЕПАТОТРОПНЫХ ЛЕКАРСТВЕННЫХ СРЕДСТВ НА ФАРМАЦЕВТИЧЕСКОМ РЫНКЕ УКРАИНЫ}

\section{(C) О. В. Геруш, О. В. Ткачева}

Цель работы: анализ ассортимента, потребления и социально-экономической доступности гепатотропных лекарственных средств (гепатопротекторов) на фармацевтическом рынке Украины за период 2012-2015 г2.

Методы исследования: мониторинг, системный и графический. Анализ ассортимента и стоимости препаратов проведен по данным системы «Фармстандарт» компании «Морион».

Результаты исследования. Анализ ассортимента и потребление гепатопротекторов показали нестабильность этого сегмента рынка и зависимость от курса доллара США, что напрямую связано с экономической ситуацией в стране и покупательной способностью населения. На протяжении 20122015 годов на рынке произошло постепенное увеличение количества недорогих отечественных гепатопротекторов и уменьшение дорогостоящих импортных препаратов. Результаты потребления гепатопротекторов в целом и в подгруппах показали, что спрос на препараты был нестабилен. С 2012 по 2013 годы в большинстве подгрупп наблюдалось значительное увеличение реализации гепатопротекторов и затем с 2013 по 2014 годы - ее снижение. Наибольший спад потребления произошел для препаратов орнитина оксоглурата (АО5В А06), у которых значительно варьирует стоимость упаковки - от 114,94 грн. (2012) до 2383,40 грн. (2015). По показателю Cа.s. в \% большинство гепатопротекторов относится к высокодоступным, что позволяет потребителям выбрать препарат по его финансовым возможностям. К малодоступным относятся в основном препараты орнитина оксоглурата и урсодезоксихолевой кислоты.

Выводы. Ассортимент гепатотропных лекарственных препаратов на фармацевтическом рынке Украины достаточен. Доля препаратов отечественного производства постепенно увеличивается. Цены на препараты в течение 2012-2015 г2. увеличились в 3-4 раза. Несмотря на рост иен до сих пор большинство гепатопротекторов являются высокодоступными для жителей Украины

Ключевые слова: хронические заболевания печени, гепатотропные лекарственные средства, ассортимент, доступность, потребление, фармацевтический рынок 